Anjou. Maine. Poitou-Charente. Touraine

108-4 | 2001

La Chambre des comptes de Bretagne

\title{
La Chambre des comptes de Jean-Baptiste Ceineray
}

\author{
Hélène Rousteau-Chambon
}

\section{(2) OpenEdition}

Édition électronique

URL : http://journals.openedition.org/abpo/1676

DOI : $10.4000 / a b p o .1676$

ISBN : 978-2-7535-1484-3

ISSN : 2108-6443

Éditeur

Presses universitaires de Rennes

Édition imprimée

Date de publication : 20 décembre 2001

Pagination : 81-98

ISBN : 978-2-86847-674-6

ISSN : 0399-0826

Référence électronique

Hélène Rousteau-Chambon, «La Chambre des comptes de Jean-Baptiste Ceineray », Annales de

Bretagne et des Pays de l'Ouest [En ligne], 108-4 | 2001, mis en ligne le 20 décembre 2003, consulté le 02 mai 2019. URL : http://journals.openedition.org/abpo/1676 ; DOI : 10.4000/abpo.1676 


\title{
La Chambre des comptes de Jean-Baptiste Ceineray
}

\author{
Hélène RouSTEAu-CHAMBON \\ Maître de conférences en histoire de l'art moderne, \\ Université de Nantes
}

La Chambre des comptes de Nantes fait figure d'exception. En effet, bien qu'elle ait changé de fonction depuis la Révolution Française (Préfecture), il s'agit de l'unique Chambre des comptes existant encore aujourd'hui, édifiée dans le but d'accueillir uniquement les conseillers ${ }^{1}$. Le seul édifice qui pouvait lui être comparé, était la Chambre des comptes de Paris, construite par Jacques V Gabriel (1667-1742) mais qui brûla en 1871. D'un point de vue historiographique, la Chambre des comptes de Nantes est donc de la plus haute importance.

Elle est tout aussi fondamentale dans la carrière de son bâtisseur, architecte-voyer de la ville de Nantes, Jean-Baptiste Ceineray. Jean-Baptiste Ceineray est né à Paris le 10 mars 1722. Il était le fils de François Ceineray, maître charpentier ${ }^{2}$. On ne connaît rien de sa formation avec certitude. Il est vraisemblable cependant que son premier apprentissage fut assuré par son père, comme cela était coutumier. Par la suite, il dut suivre l'enseignement d'un architecte de plus grand renom. J.-C. Renoul ${ }^{3}$, biographe de Ceineray, affirmait qu'il avait été formé par Jossenay, professeur à l'Académie royale d'architecture, avant d'obtenir le prix de Rome. Mais si Jossenay eut beaucoup d'élèves, le nom de Ceineray ne semble pas être mentionné et il ne remporta aucun prix.

1. Les Chambres de comptes d'Aix, de Blois, de Montpellier notamment ont été détruites; celles de Rouen, Dijon, Pau, de Montpellier... étaient installées dans des bâtiments ayant de multiples fonctions (notamment cour des Aides voire Présidial), ou éventuellement dans le château de la ville (Pau, Montpellier).

2. Arch. dép. de Loire-Atlantique, E 1330, extrait des registres de la paroisse de SaintJacques-du-Haut-Pas : « Fils de François Cenerai maistre charpentier, a été baptisé ce jour. Le parrain est Matthieu Jacob fils de mattjieu Jacob, perruquier, de la paroisse SaintBenoist et la marraine est Renne Ceinerai, sœur de l'enfant. Le parrain et la marraine ont déclaré ne savoir signer. Signé Ceinerai, Davul, vicaire. "

3. J.-C. Renoul, Ceineray, Nantes, 1862. 
Par ailleurs, Franque dans les procès-verbaux de l'Académie assurait qu'il avait été son professeur ${ }^{4}$, mais ce dernier (1710-1793) habita Avignon jusqu'en 1744 et n'entra à l'Académie, en $2^{\text {nde }}$ classe, qu'en 1754 . Il est cependant tout à fait possible que Ceineray se soit formé dans le cabinet de Franque à Paris, alors que ce dernier n'appartenait pas encore à l'Académie. Il est d'ailleurs envisageable, mais ce n'est qu'une hypothèse, que Ceineray se soit installé à Nantes, à la suite des travaux menés par Franque dans la région nantaise (Franque construisit deux " maisons de campagnes " à Nantes ${ }^{5}$ ).

Quoi qu'il en soit, le 7 mai 1754, Ceineray se maria à Nantes en l'église Saint-Nicolas avec Marie Sauvaget, fille d'un lieutenant de la milice ${ }^{6}$. Trois ans plus tard, bénéficiant sans doute de l'aide de son beau-père, il était nommé aide de Portail ${ }^{7}$, avant de lui succéder comme architecte-voyer en 1760. Son talent fut, par ailleurs, reconnu dans la capitale puisqu'il fut nommé correspondant régnicole de l'Académie en 1769. Mais sa carrière s'arrêta assez rapidement puisqu'il démissionna de son poste en $1780^{8}$ pour des raisons de santé; il avait d'ailleurs déjà été suppléé dans sa fonction d'architecte-voyer par M. Piou, architecte, le 3 mars $1768^{9}$. Ceineray mourut en 1811 dans une grande misère.

En vingt ans de carrière en tant qu'architecte-voyer de la ville de Nantes, Ceineray réalisa deux " plans d'embellissements de la ville " (1761 et 1767), une modification générale des quais sur la Loire avec l'abattement d'une partie des enceintes de la ville (quai Brancas en $1764^{10}$, quai Flesselles, 177276, place du Bouffay, projet de 1774 achevé au XIX ${ }^{\mathrm{e}}$ siècle) mais aussi des espaces liant la ville à ses faubourgs (cours Saint-André, $1774^{11}$ ) et fournit de nombreux projets urbains à l'extérieur des fortifications (place Royale, place Graslin, île Gloriette ${ }^{12}$ notamment). Il réalisa aussi, tout au long de sa carrière, un certain nombre de programmes pour des bâtiments publics, tels un théâtre et une halle aux blés (1759-1761) ${ }^{13}$, la Bourse de commerce (1769 et 1774) ${ }^{14}$, un hospice des enfants trouvés (1780) et une école de Chirurgie $(1762)^{15}$. Enfin, il construisit quelques maisons à Nantes (quai de

4. Procès verbaux de l'académie royale d'architecture, H. LEMONNIER (éd.), Paris, 1920, t. VIII, 3 mai 1773, p. 151

5. AN, 011073 179, liste des constructions que s'attribuaient Franque dans une lettre au surintendant d'Angiviller, le 5 mai 1767, dans E. BonNEL, "La chapelle Saint-Charles d'Avignon ", Congrès archéologique, 1963, p. 115-118.

6. Arch. municipales de Nantes, GG 256.

7. Une des premières mentions le concernant fait état de sa fonction d'expert pour une lanterne en 1758, Arch. dép. de Loire-Atlantique, HH 153.

8. Arch. dép. de Loire-Atlantique, BB 106.

9. Idem, FF 86 .

10. Id., D190, Arch. municipales de Nantes, II $157 \mathrm{n}^{\circ} 8$.

11. Arch. municipales de Nantes, II $160 \mathrm{n}^{\circ} 6$.

12. Idem, II $159 \mathrm{n}^{\circ} 32,1778$.

13. Id., II $158^{*}, \mathrm{n}^{\circ} 8, \mathrm{n}^{\circ} 25, \mathrm{n}^{\circ} 26$ et Arch. dép. de Loire-Atlantique, C 321.

14. Id., II $158^{*}, \mathrm{n}^{\circ} 66, \mathrm{n}^{\circ} 75, \mathrm{n}^{\circ} 79, \mathrm{n}^{\circ} 81$.

15. Cités par J.-C. Renoul, op. cit., p. 20. 
l'hôpital $1779^{16}$, boulevard Guist'hau, rue du faubourg de la Bastille ${ }^{17}$, rue Sully notamment) et remit des projets d'hôtels particuliers parmi lesquels on peut mentionner celui de l'hôtel de Belle Isle ${ }^{18}$ et celui de l'hôtel d'Aux ${ }^{19}$. Ceineray eut donc une carrière particulièrement riche dans une ville alors en pleine transformation.

La Chambre des comptes est d'autant plus importante dans la carrière de Ceineray qu'elle constitue son seul édifice public réalisé; tous les autres bâtiments sont restés à l'état de projet et ont été construits, pour une part au moins (bourse, théâtre), par son successeur Mathurin Crucy. De plus, cette Chambre des comptes est un des premiers édifices prévus par Ceineray puisque seuls les projets de théâtre et de halle au blé sont antérieurs.

Une fois rappelés ces quelques éléments biographiques nécessaires à une meilleure compréhension du sujet, je vais essayer de montrer quelles furent les conceptions architecturales de Ceineray et les influences auxquelles il fut sensible dans ce bâtiment. Il ne s'agit pas, en effet, de refaire aujourd'hui une étude précise de la Chambre des comptes de Bretagne puisqu'une analyse architecturale a déjà été menée sur le bâtiment par F. Lelièvre voici quelques années ${ }^{20}$. Je vais, en revanche, comparer l'œuvre de Ceineray d'une part avec la Chambre des comptes parisienne connue grâce à des plans et relevés, et d'autre part, avec les différents projets et réalisations architecturales de l'architecte-voyer. Enfin, pour mieux percevoir les spécificités architecturales de la Chambre des comptes les contraintes liées à la fonction du bâtiment, il faut aussi tenir compte de la production contemporaine des architectes parisiens à laquelle Ceineray pouvait être sensible. Pour ce faire et pour une plus grande clarté des propos, je n'aurais de cesse de comparer le contexte historique de l'édification des Chambres des Comptes de Paris et Nantes, avant de confronter les plans et élévations de ces édifices.

\section{Les Chambres des comptes de Paris et Nantes, leur contexte historique}

À Paris, la Chambre des comptes, la Cour des Aides, la Cour des Monnaies, le Trésor tenaient leurs assemblées dans l'enceinte du Palais, sur l'île de la Cité. La Chambre des comptes était sédentarisée depuis le $\mathrm{XIV}^{\mathrm{e}}$ siècle mais il semble qu'aucun édifice permettant d'accueillir cette seule institution n'ait été construit avant le règne de Louis XII. À cette époque, les services de la Chambre des comptes s'étaient développés et

16. Arch. municipales de Nantes, II $158^{*}, \mathrm{n}^{\circ} 17$.

17. Arch. dép. de Loire-Atlantique, D 354.

18. Arch. municipales de Nantes, II $115^{*}, \mathrm{n}^{\circ} 52$.

19. Idem, II $159, \mathrm{n}^{\circ} 48$.

20. F. Lelièvre, Nantes, La Préfecture, ancienne Chambre des comptes de Bretagne, Inventaire général des monuments et des richesses artistiques de la France, s.l., 1991. 
nécessitaient la construction de bâtiments spécifiques. C'est ainsi que Fra Giocondo $^{21}$, construisit un édifice en face de la Sainte-Chapelle en 1504. Cette construction essentiellement connue par les descriptions XVIII ${ }^{\mathrm{e}}$ siècle et par une gravure d'Israël Silvestre notamment, occupait tout le côté ouest de la cour de la Sainte-Chapelle et formait un pan coupé à l'angle de la rue de Jérusalem. Un escalier extérieur, couvert, permettait d'accéder aux antichambres qui conduisaient vers les salles d'audience ${ }^{22}$ dans ce bâtiment de plan massé. Au rez-de-chaussée se trouvaient la Chambre de France et la Chambre d'Anjou, pièces dans lesquelles travaillaient auditeurs et correcteurs. Ce bâtiment était décoré par de nombreuses sculptures et " ornements gothiques ${ }^{23}$ ", avec notamment des statues de grandeur nature, posées dans des niches et représentant Louis XII et les quatre vertus cardinales. De plus, en haut de l'escalier extérieur, au-dessus de la porte était placé un bas relief représentant un porc-épic qui portait les armes de France, accolées de deux cerfs volants; les emblèmes et la devise de Louis XII se retrouvaient par ailleurs en maints emplacements dans ce bâtiment. La Chambre des comptes de Paris était indéniablement liée à la France, et la personne royale qui avait fait construire cet édifice tenait à souligner son rôle. Nous verrons qu'à Nantes, il en était de même.

Le 27 octobre 1737, un incendie éclata et détruisit une grande partie du bâtiment et des archives. Les quelque 200 conseillers servant chaque trimestre furent alors hébergés, à partir du 5 février 1738, dans le couvent des Grands Augustins. Mais une telle installation ne pouvait être que de très courte durée puisque les religieux devaient se réunir en assemblée générale en $1740^{25}$. La reconstruction totale de la Chambre des comptes s'imposait. Elle fut très rapidement menée par le premier architecte du roi, Jacques V Gabriel, sur le même emplacement que précédemment, malgré l'étroitesse des lieux ${ }^{25}$. Les conseillers purent s'installer dans ces bâtiments dès 1740 , soit un an avant l'échéance première. Mais, peut-être à cause d'une reconstruction trop rapide (deux ans), les officiers furent confrontés à des problèmes de solidité de l'édifice qui dut être repris en $1747^{26}$. La Chambre des comptes fonctionna alors en ces lieux jusqu'en 1790. À cette date, la Chambre des comptes étant supprimée, le bâtiment fut occupé par le Bureau de la Comptabilité, puis par la nouvelle Cour des comptes à partir de 1807 et enfin par la Préfecture de Police (1842-1871). Des bâtiments furent construits dans la cour de l'ancienne Chambre et déséquilibrèrent l'édifice. Mais c'est en 1871 que le bâtiment fut complètement détruit à la

21. Fra Giovanni Giocondo (Vérone 1433-Rome 1515) appelé souvent Jean Joconde en France. Lui sont attribués en France le pont Notre-Dame et une participation au château d'Amboise et au château de Gaillon. Cet architecte vint en France à la suite de Charles VIII en 1496 avec 21 autres compatriotes (artisans, artistes et humanistes).

22. D.-A. MEnARD, Livre du centenaire de la cour des Comptes, Paris, 1907, fig. 1 : plan.

23. Piganiol DE la ForCE, Description historique de la ville de Paris, Paris, 1765, t. II, p. 38.

24. M. Gallet, Les Gabriel, Paris, 1982, p. 112.

25. Gabriel disposait d'un carré d'environ $44 \mathrm{~m}$ de côté.

26. PigANIOL DE LA ForCE, op. cit., p. 40. 
suite d'un nouvel incendie ${ }^{27}$. Il ne reste aujourd'hui plus rien de l'ancienne Chambre des comptes, hormis les projets de Jacques V Gabriel, conservés aux Archives Nationales ${ }^{28}$ et quelques gravures de qualités inégales ${ }^{29}$. Ultime question concernant le contexte historique de cet édifice : quel en fut le coût au XVIII ${ }^{\mathrm{e}}$ siècle? Gabriel avait fourni un devis estimatif qui se montait à 294326 livres en 1737. Comme souvent, les prévisions furent dépassées et la construction s'éleva à 369895 livres, 12 sols, 8 deniers ${ }^{30}$. C'est la généralité de Paris qui paya entièrement la construction du bâtiment.

La reconstruction de la Chambre des comptes de Bretagne au XVIII siècle a une tout autre origine. La Chambre des comptes de Bretagne fut installée à Nantes en 1492, par décision d'Anne de Bretagne. Dans un premier temps, de 1493 à 1496, les auditions des comptes eurent lieu dans le couvent des Cordeliers avant d'être réalisées dans une propriété ducale, la " maison de Montfort ". À la mort de Charles VIII, les officiers pensèrent pouvoir retourner à Vannes, ville plus indépendante que Nantes, mais la reine Anne envoya un nouveau mandement réaffirmant la nécessité d'installer cette institution dans la capitale des Ducs (1500). Le transfert des archives n'eut lieu que 14 ans après cette décision. Il restait à construire un bâtiment permettant d'accueillir dignement les officiers et pouvant servir de dépôt d'archives. Louis XII avait fait acheter un certain nombre de terrains (1505-1509) pour construire un édifice entre l'enceinte de la ville et le couvent des cordeliers, soit à proximité de l'Erdre, dans une zone qui était en contrebas de la ville et assez humide. Mais c'est François ${ }^{\text {er }}$ qui fit construire le bâtiment, dès 1515. Cette nouvelle Chambre formait une sorte d'enclave puisqu'une grille fermait la cour devant le bâtiment, et qu'une seule rue permettait d'y accéder. Ce bâtiment achevé en 1553 est connu par les descriptions du XVII ${ }^{\mathrm{e}}$ siècle et grâce à un lavis de Lambert Doomer ${ }^{31}$, conservée au musée Dobrée. Le bâtiment était en tuffeau, de plan massé, entre cour et jardin. Un escalier droit extérieur permettait d'accéder à la salle des procureurs, au cabinet des huissiers et au grand bureau dans lequel étaient placés les portraits des rois de France. Pour souligner l'importance symbolique et l'appartenance royale de ce bâtiment, une statue

27. Seule l'arche de Nazareth conçue vraisemblablement par Philibert de l'Orme et décorée de statues de J. Goujon, fut préservée et installée dans la cour du musée Carnavalet à Paris. Pour l'attribution à Philibert de l'Orme, $c f$. J.-M. PEROUSE DE MonTCLos, "Philibert de l'Orme à Paris ", Revue de l'art, n 114, 1996, p. 9

28. AN, N III série 106-1 à 4; E* 1146, 11 mars 1738, N III série 1074, après 1792.

29. P. P. Dubuisson, Armorial de la Chambre des Comptes de Paris où sont les noms, armes et blasons de tous nos seigneurs qui la composent..., Paris, 1761, frontispice; E. BEGUILLET, Description de l'histoire de Paris et de ses plus beaux monuments, Paris, 1779, gravure par N. Martinet.; DuRAND et JARINET, Vues pittoresques des principaux édifices de Paris, $\mathrm{n}^{\circ} 259$, Paris, 1792, gravure par Enauts-Rupilly.

30. P. BRACHET, "L'hôtel de la Chambre des comptes de Paris ", La vie urbaine, juilletseptembre 1966 , fasc. 3 , p. 14.

31. Cet artiste, peintre et dessinateur hollandais (Amsterdam, 1624-1700) avait entrepris un "Grand Tour " en Europe et se rendit à Nantes en 1646, ville dans laquelle il put rendre visite à ses deux frères, marchands et courtiers quai de la Fosse. 
équestre représentant Henri II était installée sous une arcade, au centre du bâtiment. Dans l'édifice du Xvi ${ }^{\mathrm{e}}$ siècle, tout était mis en œuvre pour souligner que la Chambre des comptes était un maillon administratif clef du royaume de France. Le roi avait fait construire ce bâtiment, il avait décidé de son emplacement, enfin, aux yeux de tous, il mettait en exergue la fonction royale avec la statue équestre en façade sur rue et les portraits des rois de France dans la salle d'audience (Grand bureau). Aucun blason ou élément décoratif ne semble, en revanche, avoir souligné que ce bâtiment était sis en Bretagne. Il s'agissait donc d'une présentation sensiblement identique à celle de la Chambre des comptes de Paris. Seule la statue équestre mettait le bâtiment au goût du jour.

$\mathrm{Au} \mathrm{XVIII}{ }^{\mathrm{e}}$ siècle, cet édifice était particulièrement vétuste; les rois de France comme le Parlement de Bretagne ne voulant vraisemblablement pas supporter la charge de son entretien. En 1750, le président de Becdelièvre voulut faire réédifier une partie du bâtiment à la suite d'une expertise menée par les maîtres-maçons Caillaud et Moreau. Mais rien ne fut alors réalisé. Il fallut attendre une nouvelle expertise (1759), menée par l'architecte voyer de la ville J.-B. Ceineray, le maître maçon Bontoux, l'ingénieur du roi, Lelièvre, et l'ingénieur des Ponts et Chaussées Villeminot, pour qu'enfin soit prise la décision de déménager le siège et les archives au couvent des Cordeliers, à cause de l'état de délabrement du bâtiment du $\mathrm{XVI}^{\mathrm{e}}$ siècle $^{32}$. Parallèlement, la construction d'une nouvelle Chambre des comptes était décidée, à proximité de celle existant jusqu'alors. Mais pour ce nouveau bâtiment, les contraintes topographiques étaient moindres puisque l'enceinte de cette partie de la ville pouvait être abattue, que l'Erdre venait d'être aménagée dans cette partie de son cours ${ }^{33}$ et que le Port communeau, port de déchargement, avait été créé.

Contrairement à la première Chambre des comptes nantaise qui était reléguée dans une partie reculée de la ville, la nouvelle construction allait être un élément clef du décor urbain. Elle était située sur le même site qu'auparavant, mais le développement de ce quartier était en cours. La façade nord du bâtiment était tournée sur l'Erdre, sa façade sud, sur une place nouvellement aménagée, et la façade est visible depuis les promenades publiques en cours d'aménagement (cours Saint-André et SaintPierre). L'insertion de la nouvelle Chambre des comptes dans le réseau urbain de la ville en plein développement était donc prévue. C'est seulement trois ans après l'expertise menée par Ceineray que fut commencée la construction de la nouvelle Chambre des comptes : les tranchées et fondations furent entreprises en mai 1762 et le bâtiment était couvert en 1769. Mais les travaux s'arrêtèrent alors, à la suite du départ du duc d'Aiguillon, gouverneur de la ville, qui avait été un fervent défenseur de ce projet et de

32. Arch. dép. de Loire-Atlantique, C 490, Correspondances sur l'état de vétusté de la Chambre des comptes, 1759-1769.

33. Les quais en cales avaient été réalisés en 1748 à la demande de l'intendant Le Bret et furent continués en 1762 sous la direction de Ceineray. 
l'opposition de la commission des États aux commissaires de la Chambre des comptes qui avaient la direction du chantier jusqu'à lors. Les travaux reprirent en 1774 mais des problèmes de stabilité de l'escalier retardèrent les opérations (nouvelles expertises de l'ingénieur Perronnet ${ }^{34}$ ). L'aménagement intérieur fut néanmoins réalisé et les conseillers purent s'y installer en 1781. Ils y restèrent pendant dix ans ${ }^{35}$. Le financement de cette nouvelle construction fut assuré pour moitié par le roi sur la coupe de bois de la forêt du Gavre, et par la province de Bretagne pour l'autre moitié ${ }^{36}$. Celleci, en effet, ne pouvait supporter la charge entière de cette construction puisqu'elle devait aussi financer la construction du Présidial de Rennes et l'amélioration de la navigation sur la Loire. Il s'agissait d'une construction prestigieuse au coût très important ${ }^{37}$. Pourtant, la ville refusa de participer à cette construction, invoquant les récentes ponctions financières du roi les précédentes années. Les conditions du financement étaient donc radicalement différentes de celles qui avaient permis la reconstruction rapide de la Chambre des comptes parisienne.

Le rappel de ces quelques éléments historiques conduit à apporter quelques comparaisons entre les deux Chambres des comptes. Au XVI ${ }^{\mathrm{e}}$ siècle, le contexte historique de la construction de ces deux bâtiments différait certes, puisqu'à Nantes, c'est le roi qui imposait une nouvelle construction dans une ville qui, jusque-là, avait rarement accueilli l'institution, mais, par ailleurs, les Chambres des comptes de Paris et de Nantes avaient une présentation sensiblement identique, François ${ }^{\text {er }}$ imposant vraisemblablement le parti parisien. Au XVIII ${ }^{\mathrm{e}}$ siècle, un certain nombre de parallèles peuvent encore être établis entre les deux édifices. En premier lieu, ces deux projets furent réalisés à 20 ans d'écart seulement, 1736-1740 pour la Chambre des comptes de Paris, 1766-1780 pour celle de Nantes. Mais, la rapidité extrême et étonnante des travaux à Paris relève certainement de la volonté royale. À Nantes, que ce soit dans le bâtiment du XVI ${ }^{\mathrm{e}}$ ou dans celui du XVIII ${ }^{\mathrm{e}}$ siècle, il n'en fut jamais de même. Le pouvoir royal imposait moins aisément ses volontés et les discordes locales ne pouvaient qu'entraver les travaux. De plus, l'opposition du Parlement au duc d'Aiguillon qui constituaient les uns et les autres, les deux soutiens majeurs pour cette construction ne pouvaient qu'entraver l'avancement de la construction. Par ailleurs, à Paris, c'est le Premier architecte du roi, alors au sommet de sa carrière, qui construisit l'édifice alors qu'à Nantes, un jeune architectevoyer qui n'avait encore rien réalisé de notable dans la ville se voyait confier le chantier. À Nantes, le statut même de l'architecte peut indiquer l'importance toute relative accordée alors à cette construction. En effet, cette construction royale peut être comparée à la réalisation des places

34. Arch. dép. de Loire-Atlantique, C 492.

35. Idem, C 493.

36. Id., C 491. Dans les années 1770, les dépenses atteignent déjà la somme de 490000 livres.

37. Pour diminuer les frais de construction, comme souvent, les matériaux de démolition provenant de l'enceinte et de l'ancien bâtiment furent utilisés. 
royales, à la très haute fonction symbolique. Or, au XVIII ${ }^{\mathrm{e}}$ siècle, ce sont essentiellement les architectes du roi, Jules Hardouin-Mansart, Robert de Cotte (Dijon), ou Jacques V Gabriel et son fils (Rennes, Bordeaux) qui furent le plus souvent appelés. La construction de telles places par des architectes locaux restait rare (Toulouse). À Nantes, ce parti peut faire référence au refus de l'interventionnisme parisien mais cette situation reflète peutêtre aussi la perte d'influence de la Chambre des comptes en tant qu'institution en Bretagne.

Outre ces éléments d'ordre historique, les deux Chambres des comptes sont comparables quant à leur implantation. La Chambre de Paris avait été installée dans un enclos " administratif " au XvI ${ }^{\mathrm{e}}$ siècle, le palais de la cité. Celle de Nantes fut, elle aussi, réalisée dans un enclos, cette fois artificiel, proche de l'Erdre. De plus, lors de sa reconstruction, la Chambre de Paris fut réédifiée sur le même emplacement, malgré le manque de place manifeste, de même que celle de Nantes, qui elle, put s'étendre. Quoi qu'il en soit, dans les deux cas, le site premier devait être conservé pour souligner la continuité de l'institution vraisemblablement, et à Paris, en outre, pour ne pas être séparée des autres institutions royales. Beaucoup de similitudes sont donc perceptibles entre ces deux organismes parisien et breton quant à leur contexte historique. Il en est de même pour le plan des édifices.

\section{Les plans}

À Paris, sur une parcelle irrégulière, puisque Gabriel devait tenir compte des bâtiments préexistants - notamment la rue de Jérusalem - l'architecte avait distribué quatre ailes élevées sur trois niveaux autour d'une cour fermée (figure $n^{\circ} 1$ ). Il avait aussi préservé un niveau de cave dans lesquels étaient conservées les archives. Gabriel avait, en fait, adopté ce type de plan pour utiliser les anciennes fondations qu'il renforça en certains points. Au rez-de-chaussée, au nord, un passage voûté permettait de faire communiquer la place de la Sainte-Chapelle à la cour. À ce niveau, quelques appartements étaient prévus dans les diverses ailes du bâtiment (portier, receveur des épices, greffier plumitif) ainsi qu'une cuisine et une buvette pour les conseillers. Mais tout le rez-de-chaussée du bâtiment principal devait accueillir les archives. Au premier étage, le bâtiment principal avait une double distribution, contrairement aux ailes en retour. Y étaient aménagées les pièces de représentation : le grand bureau, le bureau du procureur et le cabinet du Conseil. L'escalier d'honneur permettant de desservir ces espaces était rejeté dans une aile en retour et les différents accès assuraient un respect de hiérarchie en ces lieux. Dans l'aile en retour nord était installé le greffe, doté d'un petit escalier autonome. Dans l'aile sud, un couloir donnait accès au Parquet du procureur général; en outre, un escalier majestueux placé latéralement permettait d'y accéder directement. Enfin, une " galerie de communication " assurait le lien entre les deux ailes et permettait aussi d'entrer dans le dépôt d'archives (pièces voûtées d'arêtes). Ce magasin se poursuivait au troisième niveau. Au dernier étage, les 
Figure $\mathbf{n}^{\circ} 1$ La Chambre des comptes de Paris, plan de J. V Gabriel, (Arch. Nat. $\left.E^{*} 1146 A\right)$

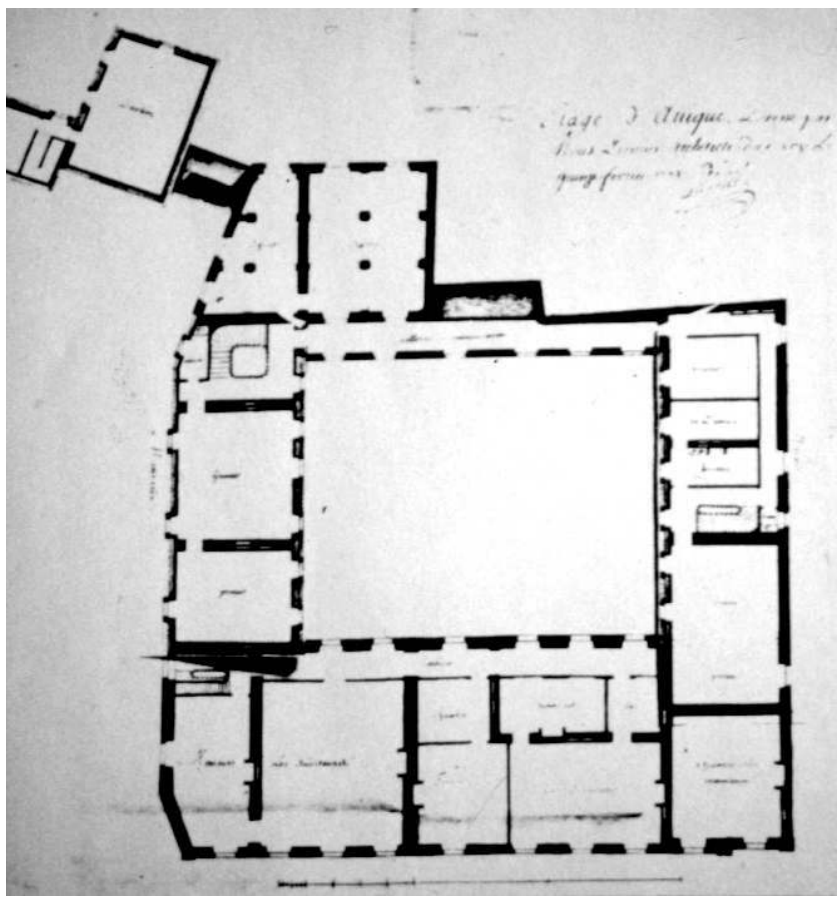

Figure $n^{\circ} 2$ La Chambre des comptes de Nantes, plan de Ceineray, (Arch. dép. de Loire-Atlantique C488)
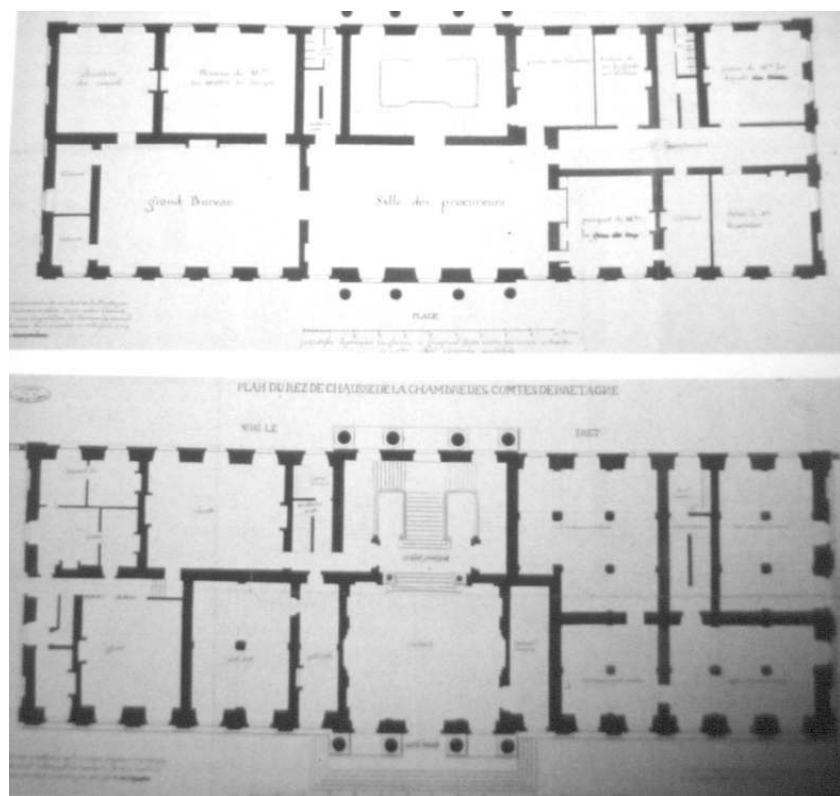
conseillers-correcteurs étaient répartis en plusieurs chambres (chambre du trésor, de la correction, de la commission, cabinet du conseiller aux fiefs). Une telle disposition des pièces, le voûtement des dépôts d'archives $^{38}$, ainsi que les nombreux escaliers desservant chaque partie de l'édifice, et le chemin de circulation montrent un souci réel d'organisation de l'espace en conformité avec la fonction du bâtiment. On peut alors être très étonné que Gabriel ait situé un bûcher sous le pavillon des archives...

La Chambre des comptes de Nantes est un édifice à deux niveaux, avec un niveau de cave dans lequel étaient entreposées les archives. De plan massé, il dispose d'une double distribution sur les deux étages (figure $n^{\circ} 2$ ). Au rez-de-chaussée, un vaste vestibule donnant sur un escalier à deux volées était placé au centre du bâtiment et ouvrait sur le petit et le grand greffe, ainsi que sur la salle des officiers dans la partie nord du bâtiment; la portion sud étant réservée à la conservation des archives. À l'étage, l'escalier menait directement dans la salle des procureurs, ouvrant elle-même d'une part sur le grand bureau et d'autre part sur le Parquet, de moindre taille que les pièces citées précédemment. Dans l'alignement de l'escalier se trouvaient aussi, au nord, le bureau des maîtres au compte et la chambre du conseil; la pièce des huissiers et celle des députés étaient au sud. Comme à Paris, on peut remarquer l'importance des escaliers qui pouvaient assurer une circulation plus aisée notamment entre les caves et le rez-de-chaussée. En outre, un escalier droit doublait l'escalier d'honneur et constituait en quelque sorte une "petite entrée " directement dans la salle des procureurs.

Dans le domaine architectural, les plans diffèrent certes entre ces deux édifices, mais cette diversité est essentiellement due aux contraintes urbaines de la capitale, les édifices publics étant, par ailleurs, le plus souvent conçus sur un plan massé. De plus, les affaires relevant de la Chambre des comptes de Bretagne étant moins importantes que celles dépendant de la Chambre des comptes de Paris, le nombre de pièces spécifiques à Nantes restait moindre (absence de petit bureau à Nantes par exemple). En outre, à Paris, Gabriel avait veillé à reprendre exactement la distribution de la Chambre des comptes du XVI ${ }^{\mathrm{e}}$ siècle dans le corps principal; à Nantes il n'en fut pas de même (pièce en enfilade $a u x{ }^{\mathrm{e}}$ siècle, distribution en double profondeur au XVIII ${ }^{\mathrm{e}}$ siècle). Mais une parenté réelle existe entre ces deux édifices quant à la distribution intérieure. Dans un cas comme dans l'autre, l'escalier d'honneur mène dans la salle des procureurs (avec une antichambre pour la Chambre des comptes de Paris). De plus, cette salle des procureurs permet d'accéder au Grand bureau à Nantes et à Paris. Seuls les emplacements de la Chambre du Conseil et du Parquet diffèrent, mais ce sont, en fait, des conséquences du plan général (massé et en U). En effet, dans les deux sites, des escaliers secondaires permettent d'accéder direc-

38. Ce voûtement avec des doubleaux semble avoir surpris les contemporains, qui, comme Piganiol de la Force, soulignèrent sa présence alors que la description du bâtiment du XVIII ${ }^{\mathrm{e}}$ siècle était fort courte. 
tement au Parquet. Dans les deux cas, une grande importance a été accordée à la " fonctionnalité " du bâtiment : voûtement des salles de conservation d'archives, circulation facilitée par les nombreux accès, distribution intérieure favorisant un accès pour certaines pièces ouvertes plus largement (greffe) et autres salles devant signifier la majesté de la fonction... Des plans logiques et cohérents dominent donc et cette distribution répond parfaitement aux missions confiées à ces Chambres des comptes. Il est vrai, en outre, que cette attention est générale au XVIII ${ }^{\mathrm{e}}$ siècle.

\section{Élévation}

Sur la place de la Sainte-Chapelle, la Chambre des comptes de Paris s'élevait sur trois niveaux, les deux premiers étant de même importance. Compte tenu du parcellaire, de l'utilisation des fondations et des nécessités d'éclairage pour la grande antichambre du premier étage, Gabriel avait dû réaliser une façade asymétrique. Mais à cause de la présence de la Sainte-Chapelle et du manque de recul sur cette place, il était impossible d'avoir une vue d'ensemble de l'édifice et donc de percevoir cette dissymétrie. L'édifice restait très sobre : l'appareil à refend soulignait les angles, la forme des fenêtres alternait en fonction des niveaux (légèrement cintré au rez-de-chaussée, linteau droit au second niveau) et les baies n'étaient décorées que d'une agrafe discrète (figure $n^{\circ} 3$ ). L'entrée du bâtiment était cependant soulignée par un avant-corps sur deux niveaux (colonnes doriques supportant un fronton droit). Les sculptures demeuraient, elles aussi, peu nombreuses : des allégories de la prudence et de

Figure $n^{\circ} 3$ - La Chambre des comptes de Paris, dessin de J. V Gabriel, (Arch. Nat. $E^{*} 1146 A$ )

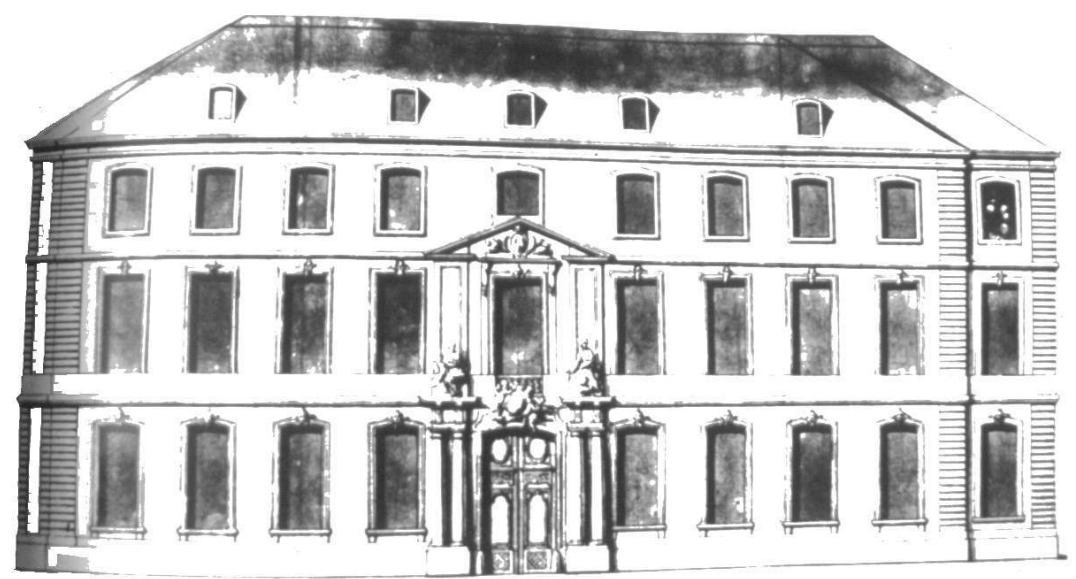


la justice d'Adam le Jeune ${ }^{39}$ surmontaient les supports, et deux blasons (dont l'un aux armes de France) soulignaient simplement la fonction de l'édifice. Côté cour, les façades étaient encore plus sobrement décorées puisque seuls des cordons moulurés soulignaient l'horizontalité du bâtiment et des agrafes décoraient la clef des arcs en plein cintre au rez-dechaussée. L'élévation n'était cependant pas identique dans les différentes ailes, indiquant ainsi une certaine hiérarchie de ces espaces puisque quatre niveaux soulignaient les ailes sud et le bâtiment des archives. Cette multitude de niveaux, cachée aux yeux des passants, indique clairement la nécessité d'utiliser le maximum d'espace, ce qui ne peut toujours être montré au nom d'une bonne esthétique architecturale. L'architecture de ce bâtiment restait donc très sobre comme dans les rares bâtiments publics construits alors. Mais ici, l'accent était mis sur l'avant-corps pour ne pas surcharger la construction. La destination de cet édifice, à la grande portée symbolique et royale, comme les goûts de Jacques V Gabriel en matière d'architecture ont certainement conduit l'architecte à ce parti de clarté et de sobriété, qu'il avait déjà mis en œuvre dans l'hôtel de ville et le Présidial à Rennes (1730).

L'élévation de la Chambre des comptes de Nantes est, quant à elle, très sobre; il s'agit d'un édifice à deux niveaux couronné d'un toit terrasse. Seul, un fronton droit supporté par des colonnes ioniques animent les volumes sur les deux façades principales (figure $n^{\circ} 4$ ). Dans le projet de Ceineray, les fenêtres à linteau droit, légèrement plus hautes au rez-de-chaussée devaient, quant à elles, être décorées de simples agrafes. Celles sur la façade principale n'ont finalement pas été réalisées. La façade " du coté de l'entrée " est cependant plus animée que celle sur l'Erdre puisque sur la première, des pilastres ioniques rythment régulièrement les travées alors que sur la deuxième, de chaînes d'angles à refend égayent seulement l'ensemble de la façade. Les façades latérales sont traitées comme des pavillons : cinq baies rythment les deux niveaux, les chaînes d'angles à refend et des chaînes de pierres à refend soulignent le portail d'entrée et la baie centrale (figure $n^{\circ} 5$ ).

Quels sont les points communs et les divergences dans l'élévation de ces deux édifices? Dans l'élévation, une très grande sobriété domine dans les deux constructions. On retrouve l'utilisation de l'appareil à refend dans les chaînes d'angle, et le décor par de simples agrafes (façade sur l'Erdre à Nantes). À Paris, le bâtiment est cependant plus élevé (3 niveaux) et le toit au comble brisé accentue cette hauteur importante. Mais là encore, les nombreuses affaires traitées comme la situation même de ce bâtiment peuvent expliquer ce parti : à Paris, il était inséré dans une masse de constructions dont il devait être distingué. Quelques différences sont aussi marquantes, notamment le traitement du toit (comble brisé à Paris, toit terrasse à Nantes). La date de construction explique ce parti. En effet,

39. Un modèle en plâtre de la justice avait été présenté lors du salon de 1739, dans J. Guifrer, Collection des livrets des salons, Paris, 1869. 
La Chambre des comptes de Jean-Baptiste Ceineray

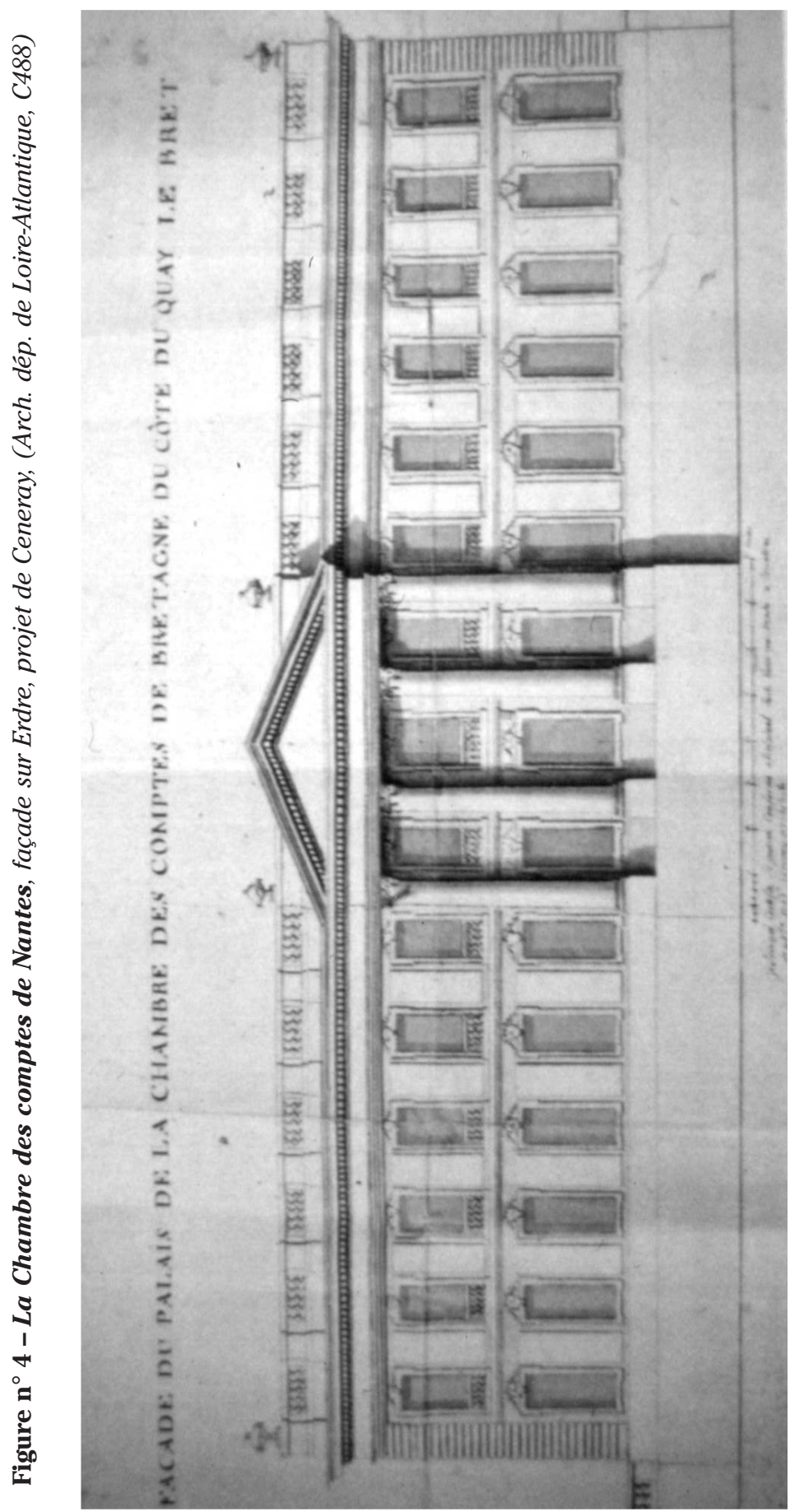


Figure $\mathbf{n}^{\circ} 5$ - La Chambre des comptes de Nantes, façade latérale depuis le cours Saint-André

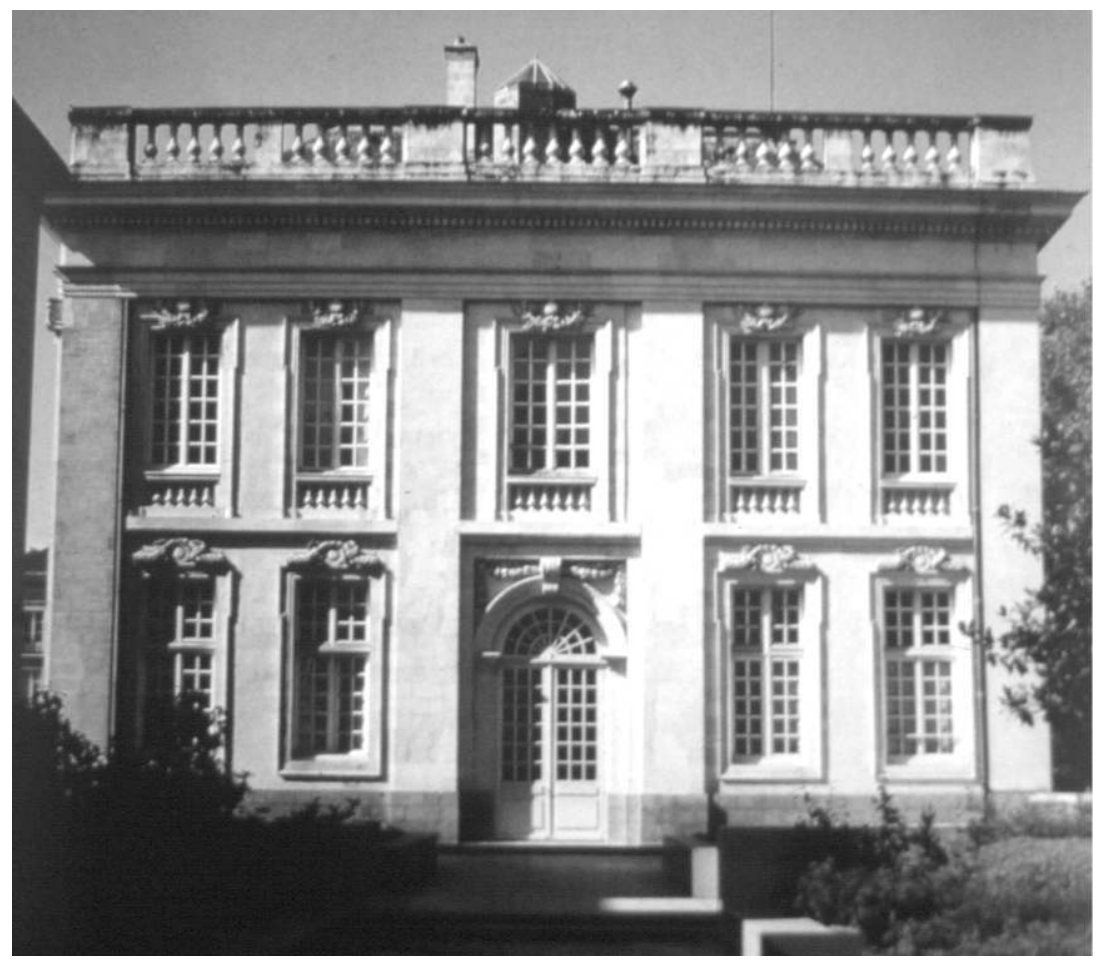

Gabriel dans la $1^{\text {re }}$ moitié du XVIII ${ }^{\mathrm{e}}$ siècle, comme bon nombre de ses contemporains, n'utilise jamais le toit terrasse mais préfère le comble brisé, mis en œuvre par son grand-oncle François Mansart. Dans la seconde moitié du XVIII ${ }^{\mathrm{e}}$ siècle, en revanche, le toit terrasse, est remis à l'honneur dans l'ensemble des constructions publiques et privées. L'autre grande différence architecturale dans ces bâtiments est le traitement de l'avant-corps. À Paris, il est sur deux niveaux - il n'embrasse donc pas l'ensemble de l'élévation -, et de faible largeur, compte tenu de la longueur de ce bâtiment (1/6 de la longueur totale). À Nantes, les proportions de cet avant-corps semblent plus harmonieuses puisqu'il fait un quart de la longueur totale. Le fait qu'il occupe toute la hauteur de la façade permet de mettre en valeur ce grand sens des proportions. Dans ces constructions, l'accent était toujours mis sur la monumentalisation de l'édifice qui était un organe de la représentation de la province lié au pouvoir royal. En dehors de cet élément clef pour tout édifice public, les besoins fonctionnels spécifiques de la Chambre des comptes n'avaient pas à paraître en façade. Aussi, les différences architecturales entre les deux Chambres des comptes reflètent, en fait, l'évolution du goût en architecture. Or, le trai- 
tement des façades par Ceineray est plus proche de celui de Ange-Jacques Gabriel et que de celui du père de ce dernier.

Dans l'élévation, en effet, excepté pour quelques détails architecturaux (chapiteaux ioniques à chutes), beaucoup de parallèles peuvent être opérés entre la Chambre des comptes et l'œuvre de Ange-Jacques Gabriel (1698-1782) qui succéda à son père en tant que Premier architecte du roi. Certes, Ange-Jacques Gabriel s'est beaucoup inspiré de l'art de son père. Mais, il a aussi été profondément marqué par l'art du Grand Siècle (place de la Concorde ou École militaire par exemple), tout en étant réceptif au nouveau goût pour le retour à l'antique dans certaines de ces réalisations (Petit Trianon). Ceineray fut sensible à ces multiples références qui se retrouvent dans la Chambre des comptes (les deux niveaux, le toit terrasse, l'avant-corps important sur une des façades, et sur l'autre les pilastres cannelés corinthiens rythmant la façade). Ce vocabulaire émane directement de celui utilisé dans le Petit Trianon (1762-1764) par exemple. Ceineray pouvait faire référence à cette œuvre, la production de l'architecte parisien étant largement diffusée par la gravure. De plus, les deux architectes eurent la possibilité de se côtoyer à Paris puisqu'ils étaient contemporains. Ceineray put aussi s'inspirer de J.-F. Blondel, comme l'avait remarqué M. Rabreau ${ }^{40}$. Blondel, en effet, avait publié des ouvrages illustrés par des gravures qui firent date, notamment, L'architecture française (1752-1756). L'auteur y développe un goût pour une certaine sobriété architecturale tout en veillant à donner une grande majesté aux édifices publics. Par ailleurs, les liens unissant l'architecte de la Chambre des comptes aux architectes parisiens, les Premiers architectes du roi (Jacques V et Ange-Jacques Gabriel) comme J.-F. Blondel indiquent nettement que Ceineray avait une connaissance directe de l'architecture royale, ce qui est aisément compréhensible compte tenu des relations qu'il entretint avec l'Académie royale d'architecture ${ }^{41}$. En fait, la Chambre des comptes reste sans conteste la construction la plus " royale " de Ceineray, si on la compare à l'ensemble de son œuvre.

Un certain nombre d'éléments architecturaux se retrouvent dans plusieurs projets et constructions de Ceineray : notamment le toit terrasse dans ses constructions publiques (Bourse et théâtre), les pilastres colossaux (Bourse, pavillon d'angle du quai Brancas, place de l'Oratoire...), le fronton droit (Bourse, place du Bouffay, quais Brancas, quai Flesselles, cour des États, place de l'Oratoire...). Dans ces quelques exemples, Ceineray se montre un digne continuateur de l'art de A.-J. Gabriel et de J.-F. Blondel. De même, Ceineray, dans la Chambre des comptes, dévoile

40. M. Rabreau, " Le néo-classicisme ", Monuments Historiques, n 108, p. 38.

41. Il envoyait régulièrement des comptes rendus de ces travaux, notamment sur la Chambre des comptes, $P V$ de l'académie royale d'architecture, H. LEMONNIER (éd.), t. VII, p. 147, 27 juin 1763 et p. 270, 23 février 1767. Ibidem, t. VIII, p. 151-152, 3 mai 1773 projets d'embellissements de la ville de Nantes, ibid., t. VIII, p. 181, 7 février 1774, sur la Bourse qui est jugée "satisfaisante ". 
un grand souci pour le détail architectural. Ainsi, des chambranles à crossettes soulignent toutes les baies dans cet édifice, tandis que sur la façade nord et sur les murs latéraux non pourvus de pilastres, des décors de feuillages, des agrafes à l'étage décorent les fenêtres. Enfin, sur la façade nord, des plates-bandes entre les fenêtres donnent plus du relief à l'ensemble. Cette décoration discrète se retrouve dans toutes ses constructions contemporaines : quai Brancas, place de l'Oratoire avec toujours les chambranles à crossettes et les agrafes voire des mascarons sur le quai Brancas (l'ensemble le plus décoré de l'architecte). Dans ses autres édifices publics (Bourse, théâtre), le décor architectural est plus sobre puisque les fenêtres étaient simplement encadrées d'un chanfrein plat (Bourse, quai Flesselles, quai de l'hôpital...).

Ces quelques éléments de décor architectural restent toujours sobres et mesurés chez Ceineray, comme le préconisaient ses maîtres à penser. Aussi, la richesse des chapiteaux ioniques dans la Chambre des comptes surprend-elle d'autant plus. En effet, non seulement des fleurs décorent l'abaque - ce qui reste assez courant - mais des chutes s'additionnent. On en retrouve aussi place de l'Oratoire. Ceineray cite ici l'œuvre de Jacques V Gabriel, qui les met en œuvre sur la place royale de Bordeaux (actuelle place de la Bourse, 1735-1745) ou sur celle de Rennes (actuelle place de l'Hôtel de ville, 1725-1735). Cette richesse décorative n'avait cependant rien à voir avec l'exubérance que l'on retrouve souvent dans les constructions des années 1730-1750 (architecture " rococo "). Par ailleurs, dans l'ensemble de ces projets de constructions publiques, Ceineray projetait des édifices de différents niveaux d'élévation : un seul pour la Bourse, deux pour la Chambre des comptes et trois pour le théâtre. Ce parti relève certainement de l'insertion des édifices dans un espace plus ou moins urbanisé, inscription à laquelle veillait toujours l'architecte nantais. Ainsi, la Chambre des comptes était-elle installée à l'extrémité d'un terre-plein et une rue devait mener vers elle; elle était donc visible de loin. Mais elle était située en contrebas de la ville, malgré le comblement opéré par Ceineray; il fallait alors tenir compte de la dénivellation et deux niveaux étaient nécessaires. En revanche, pour la Bourse, située à l'extrémité du Port au vin, perceptible de loin et de toute part, un seul niveau était indispensable. Le théâtre, quant à lui, devait, au contraire, être intégré dans un espace fortement urbanisé (autour de la place royale actuelle), Ceineray avait donc alors conçu trois niveaux. Dans tous ses projets, quelles que soient les réussites architecturales auxquelles il parvint par ailleurs, Ceineray menait donc une véritable réflexion architecturale et urbaine.

En comparant la Chambre des comptes avec les autres projets d'édifice publics de Ceineray, il devient aisé de comprendre l'importance architecturale qu'occupe cet édifice dans sa carrière. Si un certain nombre d'éléments architecturaux se retrouvent dans l'ensemble de son œuvre (constructions publiques, dans ses ensembles urbains, voire dans les édifices privés édifiés dans les années 1760-1770), la variété du vocabulaire dans 
l'élévation (appareil à refend et pilastres) permet d'éviter toute monotonie dans la Chambre des comptes. Par ailleurs, malgré cette richesse, Ceineray sait préserver une très grande sobriété dans un bâtiment aux proportions harmonieuses. Éléments que l'on retrouve partiellement dans son projet de théâtre auquel on peut cependant reprocher une absence de monumentalité. Quant à la Bourse, ses proportions sont beaucoup moins satisfaisantes que dans la Chambre des comptes ${ }^{42}$. En fait, c'est la sobriété même de la Chambre des comptes qui donne de la grandeur à cet édifice, grandeur qui va de paire avec la fonction même de l'édifice. Il est certain que ce bâtiment qui représente la fonction royale est un manifeste de cette architecture royale mise en œuvre par les Premiers architectes de Louis XV, Jacques V Gabriel et Ange-Jacques Gabriel.

Les Chambres des comptes de Paris et de Nantes peuvent être mises en parallèle tant d'un point de vue historique qu'architectural. L'influence des maîtres parisiens les plus éminents reste indiscutable dans la Chambre des comptes de Nantes. En fait, il n'est pas étonnant que Ceineray alors très jeune architecte se soit inspiré de ces illustres architectes; cette démarche devait d'ailleurs être appuyée par le duc d'Aiguillon, gouverneur de Bretagne et donc représentant du roi dans la Province. En effet, la Chambre des comptes reste un des lieux dans lequel est déléguée la puissance royale. La fonction même de la Chambre des comptes exigeait cette référence au roi, et ce d'autant plus qu'il s'agissait du seul édifice nantais montrant la puissance souveraine.

Une rapide confrontation entre les édifices publics de Ceineray permet en fait de montrer que lorsque Ceineray put s'appuyer sur l'œuvre des architectes parisiens, il parvint à une grande réussite. En revanche, lorsqu'il ne put le faire, parce que les édifices devaient mettre en avant la municipalité, il ne réussit pas à adapter son vocabulaire et à mettre en œuvre une architecture signifiante en lien avec le commanditaire. Dans l'œuvre de Ceineray, la Chambre des comptes fait donc figure d'exception à plusieurs titres. Elle apparaît véritablement comme le chef-d'œuvre de Ceineray, un coup d'éclat par un homme jeune qui sut puiser des références adéquates dans l'architecture contemporaine et faire des citations de l'œuvre de ses prédécesseurs, tout en construisant un édifice à la portée symbolique certaine.

42. Même dans ses ensembles urbains, à l'exception de la place de l'Oratoire, Ceineray a réalisé des façades très longues qu'il rythmait avec difficulté. 


\section{RESUME}

J.-B. Ceineray bâtit la Chambre des comptes de 1762 à 1781. Tout en s'inspirant des grandes réalisations contemporaines, il prit pour modèle la Chambre des comptes de Paris, reconstruite à partir de 1738 par Jacques V Gabriel. La Chambre des comptes témoigne d'une parfaite maîtrise de ces diverses références; elle constitue indiscutablement le chef d'œuvre de JeanBaptiste Ceineray.

\section{ABSTRACT}

J.-B. Ceineray builds the Chambre des comptes from 1762 to 1781 . He took his inspiration from the great contemporary creations, such as the Chambre des compte of Paris, rebuilt from 1738 by Jacques V Gabriel. The Chambre des comptes testifies to a perfect control of these various references; it indubitably constitutes the masterpiece of Jean-Baptiste Ceineray. 